\title{
Impaired in vivo mitochondrial function but similar intramyocellular lipid content in patients with type 2 diabetes mellitus and BMI-matched control subjects
}

\author{
V. B. Schrauwen-Hinderling $•$ M. E. Kooi • \\ M. K. C. Hesselink • J. A. L. Jeneson • W. H. Backes • \\ C. J. A. van Echteld • J. M. A. van Engelshoven • \\ M. Mensink • P. Schrauwen
}

Received: 12 June 2006 / Accepted: 30 August 2006/ Published online: 9 November 2006

(C) Springer-Verlag 2006

\begin{abstract}
Aims/hypothesis Mitochondrial dysfunction and increased intramyocellular lipid (IMCL) content have both been implicated in the development of insulin resistance and type 2 diabetes mellitus, but the relative contributions of these two factors in the aetiology of diabetes are unknown. As obesity is an independent determinant of IMCL content,
\end{abstract}

V. B. Schrauwen-Hinderling $(\bowtie) \cdot$ M. E. Kooi $\cdot$ W. H. Backes •

J. M. A. van Engelshoven

Department of Radiology, University Hospital Maastricht,

P.O. Box 5800, 6202 AZ Maastricht, the Netherlands

e-mail: v.schrauwen@hb.unimaas.nl

V. B. Schrauwen-Hinderling • M. K. C. Hesselink • M. Mensink •

P. Schrauwen

Nutrition and Toxicology Research Institute Maastricht,

NUTRIM, Maastricht University,

Maastricht, the Netherlands

M. Mensink $\cdot$ P. Schrauwen

Department of Human Biology, Maastricht University,

Maastricht, the Netherlands

M. K. C. Hesselink

Department of Movement Sciences, Maastricht University,

Maastricht, the Netherlands

\section{J. A. L. Jeneson}

Department of Biomedical Engineering,

Eindhoven University of Technology,

Eindhoven, the Netherlands

C. J. A. van Echteld

Department of Cardiology, University Medical Center Utrecht,

Utrecht, the Netherlands we examined mitochondrial function and IMCL content in overweight type 2 diabetes patients and BMI-matched normoglycaemic controls.

Methods In 12 overweight type 2 diabetes patients and nine controls with similar BMI $\left(29.4 \pm 1\right.$ and $29.3 \pm 0.9 \mathrm{~kg} / \mathrm{m}^{2}$ respectively) in vivo mitochondrial function was determined by measuring phosphocreatine recovery halftime (PCr half-time) immediately after exercise, using phosphorus-31 magnetic resonance spectroscopy. IMCL content was determined by proton magnetic resonance spectroscopic imaging and insulin sensitivity was measured with a hyperinsulinaemic-euglycaemic clamp.

Results The PCr half-time was $45 \%$ longer in diabetic patients compared with controls $(27.3 \pm 3.5$ vs $18.7 \pm 0.9 \mathrm{~s}$, $p<0.05)$, whereas IMCL content was similar $(1.37 \pm 0.30$ vs $1.25 \pm 0.22 \%$ of the water resonance), and insulin sensitivity was reduced in type 2 diabetes patients $(26.0 \pm 2.2$ vs $18.9 \pm$ $2.3 \mu \mathrm{mol} \mathrm{m^{-1 }} \mathrm{kg}^{-1}, p<0.05$ [all mean \pm SEM]). PCr halftime correlated positively with fasting plasma glucose $\left(r^{2}=0.42, p<0.01\right)$ and $\mathrm{HbA}_{1 \mathrm{c}}\left(r^{2}=0.48, p<0.05\right)$ in diabetic patients.

Conclusions/interpretation The finding that in vivo mitochondrial function is decreased in type 2 diabetes patients compared with controls whereas IMCL content is similar suggests that low mitochondrial function is more strongly associated with insulin resistance and type 2 diabetes than a high IMCL content per se. Whether low mitochondrial function is a cause or consequence of the disease remains to be investigated.

Keywords Diabetes $\cdot$ IMCL $\cdot$ Insulin resistance Lipid metabolism · Skeletal muscle $\cdot$ Mitochondrial function . Oxidative capacity 


$\begin{array}{ll}\text { Abbreviations } & \\ \mathrm{IMCL} & \text { intramyocellular lipids } \\ \mathrm{PCr} & \text { phosphocreatine } \\ \mathrm{P}_{\mathrm{i}} & \text { inorganic phosphate } \\ \mathrm{PCr} \text { half-time } & \text { phosphocreatine recovery half-time } \\ \mathrm{PGC} 1 \alpha & \text { peroxisome-proliferator activated receptor } \\ & \gamma \text { co-activator } 1 \alpha \\ { }^{1} \mathrm{H}-\mathrm{MRS} & \text { proton magnetic resonance spectroscopy } \\ { }^{31} \mathrm{P}-\mathrm{MRS} & \text { phosphorus-31 magnetic resonance } \\ & \text { spectroscopy } \\ { }_{V} \mathrm{O}_{2 \max } & \text { maximal oxygen uptake }\end{array}$

\section{Introduction}

As the Western lifestyle has spread globally, type 2 diabetes mellitus has become an epidemic [1]. Insulin resistance in skeletal muscle is one of the earliest hallmarks in the development of type 2 diabetes. Increased accumulation of triacylglycerols (intramyocellular lipids, IMCLs) [2, 3] and decreased levels of oxidative enzymes [3] have been reported in skeletal muscle of diabetic patients. In recent years, novel data have pointed to a central role of mitochondrial dysfunction in the development of muscular insulin resistance. It has been found that a cluster of oxidative genes under the control of PPARGC1A (also known as peroxisome-proliferator activated receptor $\gamma$ coactivator $1 \alpha[\mathrm{PGC} 1 \alpha]$ ) is reduced in the (pre-)diabetic state $[4,5]$. In addition, electron microscopy studies have revealed morphological aberrations of skeletal muscle mitochondria (mitochondrial damage and smaller mitochondrial size) in type 2 diabetic patients [6]. These data suggest that mitochondrial function may be reduced in diabetic patients. Decreased oxidative capacity due to mitochondrial dysfunction may be the reason for the accumulation of triacylglycerol and its metabolites in skeletal muscle. The accumulation of these metabolites has been suggested to impede insulin signalling [7].

With magnetic resonance spectroscopy (MRS), both mitochondrial function and IMCL content can be measured non-invasively in the same muscle [8,9]. With this technique, decreased mitochondrial function and increased IMCL content were found in healthy subjects at risk of developing diabetes (i.e. insulin-resistant offspring of type 2 diabetes patients) [8]. In addition, reduced mitochondrial function and increased IMCL content have been found in insulin-resistant elderly people [9]. The finding that these aberrations were already present in healthy offspring of type 2 diabetes patients suggests that decreased mitochondrial function and increased IMCL content may be primary risk factors for developing diabetes. However, these studies do not provide information on the relative importance of high IMCL content vs low mitochondrial function in the aetiology of diabetes. One way to further investigate the importance of mitochondrial function in the aetiology of type 2 diabetes is by comparing in vivo mitochondrial function and IMCL content in obese type 2 diabetic patients and BMI-matched normoglycaemic control subjects. This is particularly interesting because IMCL levels are already elevated in obesity $[10,11]$ and similar IMCL contents have been found in type 2 diabetes patients and BMImatched controls [12]. The question therefore remains whether obese type 2 diabetic patients can be distinguished from obese normoglycaemic controls with regard to differences in mitochondrial function. Therefore, the aim of the present study was to examine in vivo mitochondrial function under conditions of enhanced metabolic demand and IMCL content in type 2 diabetes patients and BMImatched controls.

\section{Subjects and methods}

Twelve male type 2 diabetes patients and nine male BMImatched control subjects participated in the study. Control subjects had normal glucose tolerance, as measured with an OGTT, and had no family history of diabetes. Diabetic subjects had been diagnosed at least 1 year before inclusion, and had well-controlled type 2 diabetes. All type 2 diabetes subjects were on oral antidiabetic medication (five subjects on metformin, four subjects on sulphonyl ureas, two subjects on metformin and sulphonylureas and one subject on thiazolidinediones). Subjects did not perform regular intensive exercise nor had they followed a strict diet or weight reduction programme in the last year before inclusion. The protocol was approved by the medical ethics committee of the University Hospital Maastricht and Maastricht University, and every subject signed informed consent after the procedures had been explained. Subjects were recruited by advertisement in local newspapers and in the university and hospital grounds.

\section{Experimental protocol}

Prior to inclusion, all subjects underwent a medical screening that included assessment of the subject's medical history and a physical examination. Besides diabetes, subjects had no other health problems and did not use any medication that could interfere with the results of the study.

Control subjects underwent a glucose tolerance test to ensure normal glucose tolerance. Two weeks prior to the experimental measurements, diabetic patients stopped their anti-diabetic medication. 
As part of the experiment, the following parameters were compared in type 2 diabetic patients and their BMI-matched controls: maximal oxygen uptake, insulin sensitivity, IMCL content, the phosphocreatine recovery half-time ( $\mathrm{PCr}$ halftime) after exercise in the vastus lateralis muscle, and muscular $\mathrm{pH}$ at the end of exercise. A fasting blood sample was taken to determine glucose and (in diabetic patients only) glycosylated haemoglobin $\left(\mathrm{HbA}_{1 \mathrm{c}}\right)$ concentrations.

\section{Glucose standard test}

A standard oral glucose tolerance test according to WHO criteria was performed in control subjects.

Maximal oxygen uptake (whole-body oxidative capacity)

A routine incremental cycling test was used to determine the maximal aerobic capacity, as described earlier [13]. Oxygen consumption was measured continuously throughout the test using indirect calorimetry (Oxycon Beta; Mijnhardt, the Netherlands) in order to determine maximal oxygen uptake $\left(V \mathrm{O}_{2 \max }\right) \cdot V \mathrm{O}_{2 \max }$ was determined in all subjects except for three diabetic patients.

Insulin sensitivity

Insulin sensitivity was measured with a 3 -h hyperinsulinaemic-euglycaemic clamp according to DeFronzo et al. [14]. Subjects were asked to keep a constant eating pattern and to refrain from intense physical exercise during the last 3 days before the clamp. After an overnight fast, subjects were infused with insulin at a fixed rate $\left(40 \mathrm{mU} \mathrm{m}^{-2} \mathrm{~min}^{-1}\right)$. Glucose was infused at a variable rate in order to maintain euglycaemia. Insulin sensitivity is expressed as the glucose infusion rate during euglycaemia $\left(\mu \mathrm{mol} \mathrm{kg} \mathrm{min}^{-1}\right)$.

\section{MRS measurements}

Measurements were performed with a $1.5 \mathrm{~T}$ whole-body scanner (Intera; Philips Medical Systems, Best, the Netherlands) in the afternoon and subjects were asked to consume lunch $2 \mathrm{~h}$ beforehand.

\section{${ }^{31} \mathrm{P}-\mathrm{MRS}$}

A $6 \mathrm{~cm}$ surface coil was used for localisation and was fixed in the middle of the vastus lateralis muscle. First, a nonsaturated spectrum was acquired (repetition time [TR] $20 \mathrm{~s}$, 16 measurements, spectral bandwidth $1,500 \mathrm{~Hz}$, adiabatic pulse, $90^{\circ}$ pulse angle, four phase cycling steps; see Fig. 1 for typical example). Subsequently, a series of 180 partially saturated spectra was acquired $(\mathrm{TR}=4 \mathrm{~s}$, one measurement, spectral bandwidth $1,500 \mathrm{~Hz}$, adiabatic pulse, $90^{\circ}$ pulse

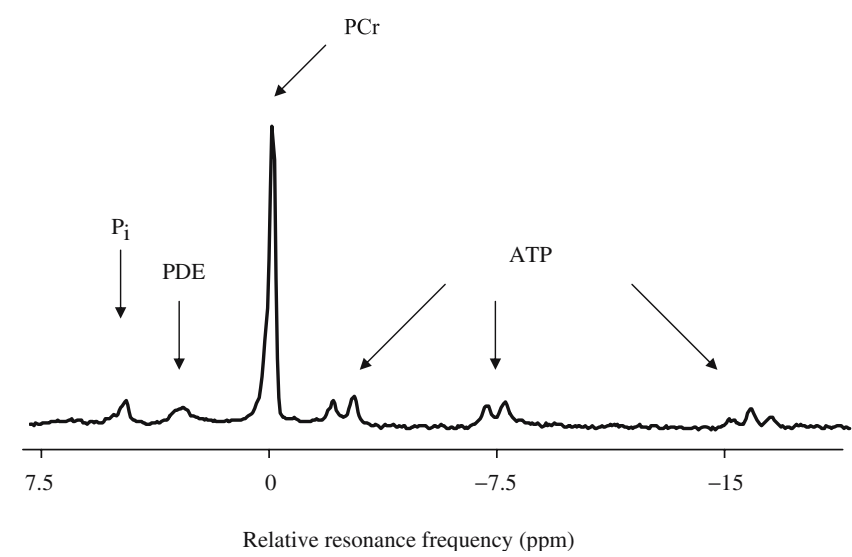

Fig. 1 Typical ${ }^{31} \mathrm{P}-\mathrm{MR}$ spectrum of the vastus lateralis muscle at rest (post-processed with $5 \mathrm{~Hz}$ Lorentzian line broadening). The arrows indicate inorganic phosphate $\left(\mathrm{P}_{\mathrm{i}}\right)$, phosphodiester (PDE), creatine phosphate (PCr) and ATP

angle). The 12 min acquisition time of the time series was structured as follows: 2 min of rest, 5 min of knee extension exercise and $5 \mathrm{~min}$ of recovery. The knee extension exercise was performed at $0.5 \mathrm{~Hz}$ to an acoustic cue on a home-built MR-compatible ergometer with a pulley system. The exercise was performed with a weight corresponding to $50-60 \%$ of predetermined maximal capacity. The maximal capacity was determined beforehand (on a different day) in an incremental maximal test with the same ergometer.

Post-processing ${ }^{31} \mathrm{P}-\mathrm{MRS}$

Peak fitting After application of phase correction and line broadening (and DC correction for the spectra of the time series), spectra were fitted in the time domain with the AMARES algorithm in jMRUI software (http://www.mrui. uab.es) using prior knowledge [15].

Prior knowledge was applied with constraints for the line widths and frequencies. In the non-saturated spectra, the inorganic phosphate $\left(\mathrm{P}_{\mathrm{i}}\right) / \mathrm{PCr}$ ratios were calculated. In four subjects, repeated measurements on different days were performed to assess the reproducibility of the $\mathrm{P}_{\mathrm{i}} / \mathrm{PCr}$ ratio. The coefficient of variation was $6.0 \pm 3.1 \%$.

Five peaks were fitted with Gaussian curves $\left(\mathrm{P}_{\mathrm{i}}, \mathrm{PCr}\right.$ and three ATP peaks) in the partially saturated spectra of the time series. In the partially saturated spectra, prior knowledge was applied also to the relative amplitudes of the three ATP peaks, taking into account the different $\mathrm{T} 1$ relaxation times [16]. To determine the $\mathrm{pH}$ during the exercise period, five consecutive spectra of the time series were added and the $\mathrm{pH}$ was calculated in $\mathrm{jMRUI}$ from the frequency shift between the $\mathrm{PCr}$ and $\mathrm{P}_{\mathrm{i}}$ peaks.

Analysis of time-course of $\mathrm{PCr}$ The time-course of the $\mathrm{PCr}$ amplitude $[\operatorname{PCr}(t)]$ during the last $20 \mathrm{~s}$ of exercise (steady state) and during the recovery period was fitted to the 
following formulas with MATLAB software (Mathworks, Natick, MA, USA) using the Levenberg-Marquardt optimisation algorithm:

before $t_{0}: \operatorname{PCr}(t)=\operatorname{PCr}\left(t_{0}\right)$

after $t_{0}: \operatorname{PCr}(t)=\operatorname{PCr}\left(t_{0}\right)+D\left(1-e^{-k\left(t-t_{0}\right)}\right)$

where $t_{0}$ is the end-time of exercise, $D$ is the difference between steady state and the $\mathrm{PCr}$ level after recovery, $\operatorname{PCr}\left(t_{0}\right)$, is the $\mathrm{PCr}$ content in steady state during exercise, $k$ is the rate constant of $\mathrm{PCr}$ resynthesis (unit, $1 / \mathrm{s}$ ). Estimates of $t_{0}, D$, $\operatorname{PCr}\left(t_{0}\right)$ and $k$ were calculated using the fitting procedure.

From the fitted curve, the PCr half-time $\left(t_{1 / 2}\right)$ was determined according to $t_{1 / 2}=\ln 2 / k$ (see Fig. 2 for a typical example).

The reproducibility of $\mathrm{PCr}$ half-time was determined by assessing this parameter twice on different days in three subjects. The mean CV of PCr half-time was $6.9 \pm 0.8 \%$.

\section{${ }^{1} \mathrm{H}-\mathrm{MRSI}$}

Image-guided ${ }^{1} \mathrm{H}-\mathrm{MR}$ spectroscopic imaging was performed in the vastus lateralis muscle after the assessment of in vivo mitochondrial function. A flexible surface coil was wrapped around the upper leg and T2 images were acquired as described earlier [17]. A point-resolved spectroscopy (PRESS) box was placed to cover the whole vastus lateralis muscle and outer volume suppression was used to suppress the signal of subcutaneous adipose tissue and bone marrow. ${ }^{1}$ H-MRSI (matrix size, $20 \times 20$ ) was used

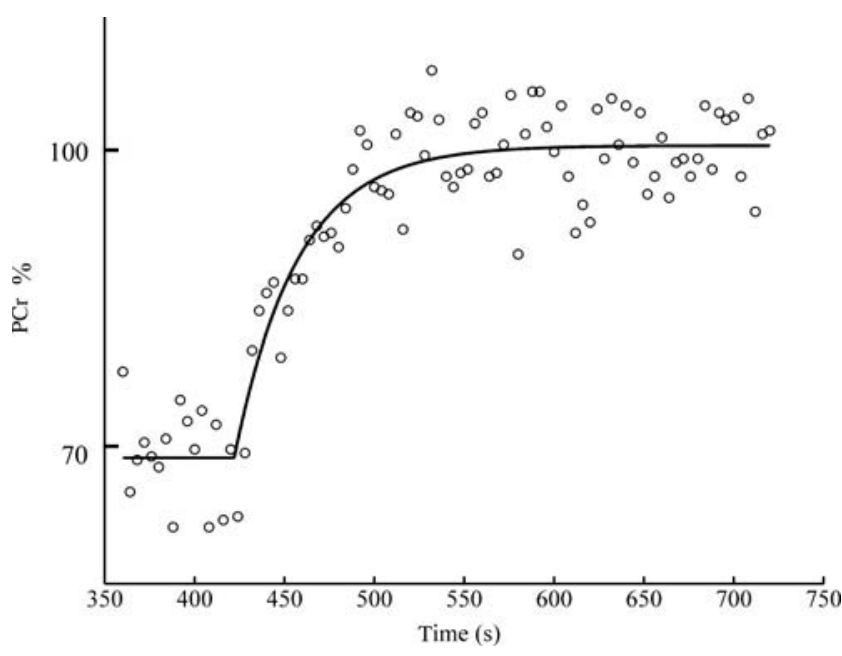

Fig. 2 Typical PCr recovery of an individual patient, with monoexponential growth preceded by the steady state fitted to the measured data $(\mathrm{PCr}$ half-time $=24.9 \mathrm{~s})$ with the following parameters: field of view, $100 \times 100 \mathrm{~mm}$; slice thickness, $18 \mathrm{~mm}$; number of signal averages, 2; repetition time, $1,500 \mathrm{~ms}$; echo time, $24 \mathrm{~ms}$; 1,024 data points over $1,000 \mathrm{~Hz}$ spectral width. This resulted in a nominal voxel size of $5 \times 5 \times 18 \mathrm{~mm}$. The water signal was suppressed using chemically selective saturation (CHESS). The unsuppressed water signal was acquired with identical resolution and was used for $\mathrm{B}_{0}$ correction and as a reference signal.

\section{Post-processing of ${ }^{1} \mathrm{H}-\mathrm{MRSI}$}

Two-dimensional Fourier transformation was applied to the water-suppressed and non-suppressed measurements, using spatial apodisation (Hanning filter, 0.25 width) and a digital shift accumulation (DSA) filter to remove the residual water signal. Voxel positions inside the vastus lateralis muscle were identified on the T2-weighted images and the corresponding spectra were inspected with dedicated software (SI-View; Institute of Biomedical Engineering (IBE), University and ETH Zurich, Switzerland). The vastus lateralis muscle was subdivided into three anatomical regions (lateral, middle and medial). Since it is known that regional variations in fibre type distribution [18] and consequently IMCL content can occur within a single muscle, only spectra from the middle region were included. All spectra with at least slight separation of the IMCL and IMCL peak were fitted using the jMRUI package fitted in the time domain using a non-linear least-squares algorithm (AMARES [19]) as previously described [17]. An experienced spectroscopist who was blinded to the origin of the spectrum performed quality ratings on all the spectra using the categories 'excellent', 'good', 'reasonable', 'moderate' and 'bad'. Only spectra of good or excellent quality were included. On average, $2.9 \pm 0.6$ spectra per subject were included, originating from the middle anatomical region in all subjects. Inclusion of the moderate quality spectra did not change the results. The T1- and T2-corrected amplitudes of the $\mathrm{CH}_{2}$ peak of IMCL were expressed relative to the corrected amplitude of the water peak (see Fig. 3 for a typical example). The IMCL content was determined for nine control subjects and ten type 2 diabetes patients. Because of insufficient quality of spectra in three cases, according to the criteria described above, IMCL values are reported for seven control subjects and nine patients.

\section{Analyses}

Blood analyses For the determination of fasting plasma glucose, blood was collected in tubes containing sodium fluoride $(\mathrm{NaF})$. Plasma glucose was determined using the glucose oxidase method with the Synchron LX System 


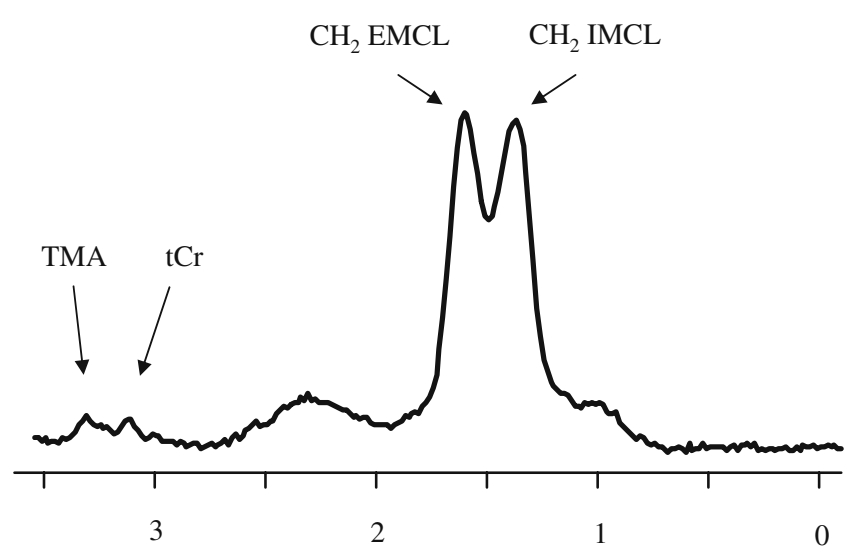

Relative resonance frequency (ppm)

Fig. 3 Typical ${ }^{1} \mathrm{H}-\mathrm{MR}$ spectrum of the vastus lateralis (postprocessed with $1 \mathrm{~Hz}$ Lorentzian line broadening). Arrows indicate peaks of trimethylammonium compounds (TMA) and total creatine $(\mathrm{tCr})$, the peak of the methylene protons of EMCL and the peak of the methylene protons of IMCL

(Beckman Coulter, Fullerton, CA, USA). Glycosylated haemoglobin was determined in a fasting plasma sample with HPLC, using Variant II (Bio-Rad, Hercules, CA, USA) and reference values of $4.4-6.2 \%$.

During the clamp, blood was collected in EDTA-containing tubes, and plasma glucose was determined with a standard enzymatic technique automated on the Cobas Fara centrifugal analyser (Glucose HK 125; ABX Diagnostics, Montpellier, France). Insulin concentration was determined using an RIA (Linco Research, St Charles, MO, USA).

\section{Statistics}

All results are reported as mean \pm SEM. Statistical analyses were performed with SPSS for Windows 11.5 software (SPSS Inc., Chicago, IL, USA). Differences between groups were determined with two-sided unpaired Student's $t$ tests. To evaluate the relationship between variables, Pearson correlation coefficients were calculated. Results were considered significant if $p<0.05$.

\section{Results}

\section{Subject characteristics}

Type 2 diabetic patients and healthy controls were overweight males between 45 and 70 years of age, and the two groups were carefully matched for BMI $(29.4 \pm 1.0$ and $29.3 \pm 0.9 \mathrm{~kg} / \mathrm{m}^{2}$ respectively, $p=\mathrm{NS}$ ). For the diabetic and control subjects, age was $61.8 \pm 1.3$ and $56.1 \pm 2.3$ years $(p<0.05)$ and $V \mathrm{O}_{2 \max }$ was $30.6 \pm 1.8$ and $34.6 \pm 1.6 \mathrm{ml}$ $\min ^{-1} \mathrm{~kg}^{-1}(p=\mathrm{NS})$. In the diabetic subjects, the mean duration of diabetes was $6.3 \pm 5.4$ years.

Insulin sensitivity and insulin and plasma glucose concentrations

The mean glucose infusion rate was lower in type 2 diabetes patients $\left(18.9 \pm 2.3 \mu \mathrm{mol} \mathrm{min}{ }^{-1} \mathrm{~kg}^{-1}\right)$ than in controls $(26.0 \pm$ $2.2 \mu \mathrm{mol} \mathrm{min}{ }^{-1} \mathrm{~kg}^{-1}, p<0.05$ ).

Fasted plasma glucose was higher in type 2 diabetes patients than in controls $(9.6 \pm 0.6$ and $5.7 \pm 0.1 \mathrm{mmol} / 1$ respectively, $p<0.01$ ) and insulin levels were similar in the two groups $(16.1 \pm 2.0$ and $12.1 \pm 0.9 \mathrm{mU} / 1$ respectively, $p>0.05$ ). Mean $\mathrm{HbA}_{1 \mathrm{c}}$ concentration in type 2 diabetes patients was $7.3 \pm 0.3 \%$.

IMCL content

IMCL content was not different between the type 2 diabetic patients and control subjects $(1.37 \pm 0.30$ and $1.25 \pm 0.22 \%$ of the water reference signal in type 2 diabetes patients and controls respectively, $p=\mathrm{NS}$ ).

In vivo mitochondrial function

During the exercise protocol, the PCr levels decreased initially until a steady state was reached after 2-3 $\mathrm{min}$. Steady-state PCr levels were similar in the two groups $(73.0 \pm 2.1$ and $71.7 \pm 2.9 \%$ of the baseline value for the diabetic and control subjects respectively, $p=\mathrm{NS}$ ). None of the subjects showed substantial acidification during the exercise protocol; the $\mathrm{pH}$ decreased to the same amount in both groups, from $7.14 \pm 0.01$ in the resting condition to $7.10 \pm 0.01$ at the end of the exercise period (change in $\mathrm{pH}$ was 0.04 in both groups, $p<0.05$ ). The end-exercise $\mathrm{pH}$ ranged from 7.05 to 7.14 .

Mean PCr recovery half-time was $45 \%$ longer in the type 2 diabetes group than in the controls $(27.3 \pm 3.5$ vs $18.7 \pm 0.9 \mathrm{~s}$, $p<0.05$; Fig. 4), indicating impaired in vivo mitochondrial function in the type 2 diabetic patients. The $\mathrm{PCr}$ half-time correlated positively with $\mathrm{HbA}_{1 \mathrm{c}}\left(r^{2}=0.48, p<0.05\right)$ and with fasting plasma glucose $\left(r^{2}=0.42, p<0.01\right.$; Fig. 5) in type 2 diabetes patients. The latter correlation was also present in the entire group $\left(r^{2}=0.47, p<0.01\right)$. PCr halftime also correlated with whole-body oxidative capacity $\left(\dot{V} \mathrm{O}_{2 \max } / \mathrm{kg}\right)\left(r^{2}=0.49, p<0.01\right)$ in the entire group, but no correlation was found between $\mathrm{PCr}$ half-time and age $\left(r^{2}=0.06, p=\mathrm{NS}\right)$.

No differences were observed between type 2 diabetes patients and controls in resting $\mathrm{P}_{\mathrm{i}} / \mathrm{PCr}(0.13 \pm 0.01$ and $0.12 \pm 0.01 ; p=\mathrm{NS}$ ). 


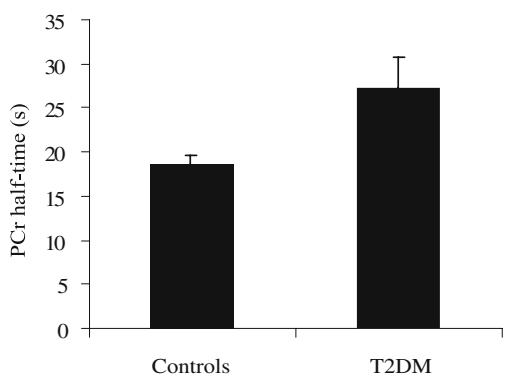

Fig. $4 \mathrm{PCr}$ half-time was longer in patients with type 2 diabetes mellitus (T2DM) than BMI-matched controls $(p<0.05)$

\section{Discussion}

Decreased mitochondrial function and therefore low oxidative capacity have been implicated in the development of insulin resistance. It has been suggested that impaired mitochondrial function leads to the accumulation of IMCL and lipid metabolites in skeletal muscle, and the latter may interfere with insulin signalling [8]. Here, we determined skeletal muscle oxidative capacity by investigating $\mathrm{PCr}$ recovery kinetics and IMCL content in the vastus lateralis muscle of type 2 diabetes patients and BMI-matched normoglycaemic controls. Interestingly, we found that $\mathrm{PCr}$ half-time was prolonged in diabetic patients, suggesting impaired mitochondrial function, whereas IMCL content was not different between type 2 diabetic patients and BMImatched control subjects. The present data fit well with the hypothesis that mitochondrial function is an important player in the development of type 2 diabetes mellitus. The strong correlation of plasma glucose concentrations as well as $\mathrm{HbA}_{1 \mathrm{c}}$, a long-term marker for glycaemic control, with $\mathrm{PCr}$ half-time may suggest that the impairment in in vivo mitochondrial function in type 2 diabetic patients is related to the degree of illness.

Decreased mitochondrial function has also been reported in insulin-resistant offspring of type 2 diabetes patients by Petersen et al. [8] when measuring in vivo mitochondrial function by assessing saturation transfer between inorganic phosphate and ATP in the resting soleus muscle. Here we

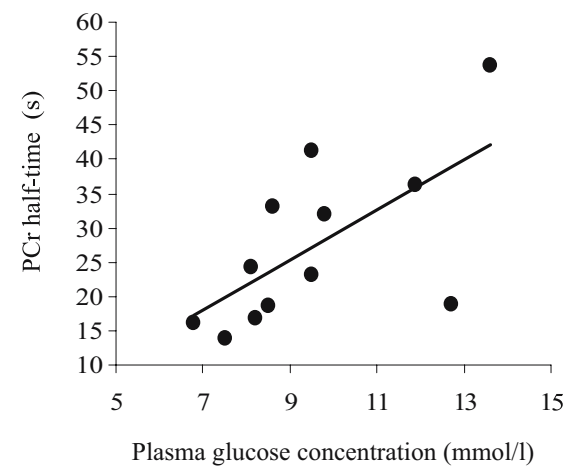

Fig. 5 Plasma glucose concentration correlates with the $\mathrm{PCr}$ half-time in diabetes patients $\left(r^{2}=0.42, p<0.01\right)$ employed an alternative ${ }^{31} \mathrm{P}-\mathrm{MRS}$ method to investigate in vivo mitochondrial function by measuring the $\mathrm{PCr}$ kinetics during recovery from exercise [20, 21]. $\mathrm{PCr}$ content decreases transiently during exercise and recovers rapidly after exercise. In the post-exercise state, $\mathrm{PCr}$ resynthesis is driven almost purely oxidatively [22] and the resynthesis rate reflects in vivo mitochondrial function [23]. Indeed, post-exercise $\mathrm{PCr}$ resynthesis has been shown to be delayed in various known mitochondrial disorders (for reviews see $[24,25])$. Furthermore, correlations of post-exercise $\mathrm{PCr}$ resynthesis kinetics and in vitro measures of mitochondrial function have been reported in healthy [26] and diabetic [27] subjects. An advantage of this in vivo method is that mitochondrial function can be assessed under conditions of increased metabolic demand. Our results under these conditions extend the findings of Petersen et al. [8], as we report a longer $\mathrm{PCr}$ half-time in type 2 diabetes patients, indicating reduced in vivo mitochondrial function, in patients with overt type 2 diabetes.

An interesting aspect of the present study is that, despite a decrease in in vivo mitochondrial function, IMCL content was not different between overweight type 2 diabetic patients and their BMI-matched controls. This suggests that a low level of mitochondrial function is a more important factor in the aetiology of diabetes than a high IMCL content. In this context, it is interesting to note that endurance-trained athletes also have high IMCL levels but are among the most insulin-sensitive subjects. In general, this apparent paradox is explained by the high muscular oxidative capacity of athletes. Our data are in line with this idea and suggest that high levels of IMCL are only associated with type 2 diabetes if combined with a low oxidative capacity, as reflected by prolonged $\mathrm{PCr}$ recovery kinetics. The mechanism by which low oxidative capacity could lead to type 2 diabetes may involve the accumulation of fatty acid metabolites such as diacylglycerol and fatty acylCoA, which have been causally related to insulin resistance. These metabolites may be specifically elevated when oxidative capacity is reduced. Indeed, together with impaired in vivo mitochondrial function, insulin sensitivity was lower in diabetic patients than in the normoglycaemic overweight control subjects.

Although the accumulation of fatty acid metabolites due to low oxidative capacity could be an explanation for the involvement of mitochondrial function in the development of type 2 diabetes mellitus, we cannot draw conclusions with respect to a causal relationship between impaired mitochondrial function and type 2 diabetes mellitus from the present study and with the current study design. Our finding that mitochondrial function is related to plasma glucose and $\mathrm{HbA}_{1 \mathrm{c}}$ levels may indeed suggest that the severity of the impairment of mitochondrial function is a determining factor for the severity of the disease. Alterna- 
tively, the reduced mitochondrial function may be the result of the insulin-resistant or hyperglycaemic state. In this context, the finding that insulin-stimulated mitochondrial ATP production is diminished in type 2 diabetic patients compared with healthy subjects [28] suggests that resistance to the action of insulin may lead to mitochondrial dysfunction. Similarly, hyperglycaemia may affect mitochondrial function negatively, as it has been shown that normalisation of high plasma glucose concentrations in diabetic patients by insulin treatment also normalises the expression of genes involved in mitochondrial bioenergetics [29]. Therefore, our finding that mitochondrial function correlated strongly with plasma glucose levels may also indicate that hyperglycaemia and/or insulin resistance is responsible for the reduction in mitochondrial function observed in our group of type 2 diabetic patients.

On the other hand, however, the results obtained from a study in first-degree relatives of type 2 diabetes patients, who have an increased risk of developing diabetes later in life, favour the idea that mitochondrial dysfunction precedes the deterioration of plasma glucose concentrations. This study shows that in vivo mitochondrial function is already decreased in healthy, normoglycaemic, insulinresistant subjects at high risk of developing type 2 diabetes mellitus [8], suggesting that mitochondrial dysfunction is a cause rather than an effect of diabetes mellitus. However, although these subjects were normoglycaemic, they were also insulin-resistant, leaving the possibility that insulin resistance may have led to reduced mitochondrial function. Therefore, the cause vs consequence issue when considering mitochondrial function and type 2 diabetes mellitus still needs further investigation. Future studies in which mitochondrial function is measured after hyperglycaemia and insulin resistance have been improved by (pharmaceutical) treatment may help to clarify this issue.

In addition, the exact mechanism responsible for the reduced in vivo mitochondrial function observed in (pre) diabetic patients is currently under debate. Two independent studies have found that a cluster of oxidative genes under the control of PGC1 $\alpha 1$, as well as the expression of PGC1 $\alpha$ itself, is reduced in patients with type 2 diabetes mellitus [5] and in subjects at high risk of developing type 2 diabetes later in life [4]. Furthermore, smaller and damaged mitochondria have been reported in the diabetic state [6]. In addition, it has recently been found that mitochondrial density, but not $\mathrm{PGC} 1 \alpha$, is decreased in insulin-resistant subjects at high risk of developing type 2 diabetes mellitus [30]. These results may point to a decreased mitochondrial content playing a role in mediating decreased mitochondrial function. In this context it is important to note that we recently found that mitochondrial protein content did not differ between patients with type 2 diabetes and BMImatched controls when a population similar to that participating in the current study was investigated [31]. Alternatively, the determination of mitochondrial function in vivo, as in the present study, can also be limited by an impairment in oxygen supply, which can occur, for example, when perfusion is disturbed. It is known that impaired perfusion is not uncommon in type 2 diabetic patients; however, we report only a very modest change in $\mathrm{pH}$ during the exercise protocol, which was identical in both groups, whereas acidification of tissue during exercise would be expected when the oxygen supply is a limiting factor. Although these results may point away from an important role of impaired perfusion in explaining the present results, it is clear that further studies are needed to elucidate the exact mechanism leading to reduced in vivo mitochondrial function in the diabetic state.

An age-related decline in mitochondrial function has been reported [9]: a difference in age of 46 years was accompanied by a $40 \%$ decline in mitochondrial function. Another study found no decline in in vivo oxidative function between the ages of 20 and 70 years; in the oldest group of subjects, who were 70-83 years old, oxidative function was decreased by $30 \%$ compared with subjects who were 50 years younger [32]. In the present study, there was an age difference of 6 years between the control and the type 2 diabetic population, and a $45 \%$ drop in mitochondrial function was found. Considering the above, we think that it is far more likely that this difference in mitochondrial function between the control subjects and the type 2 diabetics is related to the diseased state rather than to age.

In conclusion, we have shown that $\mathrm{PCr}$ half-time was prolonged in diabetic patients when compared with BMImatched normoglycaemic control subjects, which suggests that in vivo mitochondrial function is impaired in type 2 diabetes mellitus. This extends previous findings of reduced mitochondrial function in prediabetic states. Importantly, $\mathrm{PCr}$ half-time correlated positively with plasma glucose concentration in the diabetic population, revealing that the decrease in oxidative capacity is related to the degree of the disease. Whether a low oxidative capacity aggravates the disease or whether hyperglycaemia and/or insulin resistance hampers mitochondrial function is still unclear. The lower in vivo mitochondrial function in type 2 diabetes patients was not accompanied by a difference in IMCL content between the two groups, suggesting that low oxidative capacity is more closely related to muscular insulin resistance and type 2 diabetes mellitus than IMCL content per se.

Acknowledgements We thank J. van Diepen and E. Peeters for their practical help in performing the study. We also thank T. Lange and U. Dydak (Institute of Biomedical Engineering, University and ETH Zurich, Switzerland) for providing the SI-View software. P. Schrauwen is supported by a fellowship of the Royal Netherlands Academy of Arts and Sciences. M. K. C. Hesselink is supported by a 
VIDI Research Grant for innovative research from the Netherlands Organization for Scientific Research (Grant 917.66.359).

Duality of interest The authors declare that they have no duality of interest regarding this manuscript.

\section{References}

1. Zimmet P, Alberti KG, Shaw J (2001) Global and societal implications of the diabetes epidemic. Nature 414:782-787

2. Goodpaster BH, He J, Watkins S, Kelley DE (2001) Skeletal muscle lipid content and insulin resistance: evidence for a paradox in endurance-trained athletes. J Clin Endocrinol Metab 86:5755-5761

3. He J, Watkins S, Kelley DE (2001) Skeletal muscle lipid content and oxidative enzyme activity in relation to muscle fiber type in type 2 diabetes and obesity. Diabetes 50:817-823

4. Patti ME, Butte AJ, Crunkhorn S et al (2003) Coordinated reduction of genes of oxidative metabolism in humans with insulin resistance and diabetes: Potential role of PGC1 and NRF1. Proc Natl Acad Sci USA 100:8466-8471

5. Mootha VK, Lindgren CM, Eriksson KF et al (2003) PGC-1alpharesponsive genes involved in oxidative phosphorylation are coordinately downregulated in human diabetes. Nat Genet 34: 267-273

6. Kelley DE, He J, Menshikova EV, Ritov VB (2002) Dysfunction of mitochondria in human skeletal muscle in type 2 diabetes. Diabetes 51:2944-2950

7. Shulman GI (2000) Cellular mechanisms of insulin resistance. J Clin Invest 106:171-176

8. Petersen KF, Dufour S, Befroy D, Garcia R, Shulman GI (2004) Impaired mitochondrial activity in the insulin-resistant offspring of patients with type 2 diabetes. N Engl J Med 350:664-671

9. Petersen KF, Befroy D, Dufour S et al (2003) Mitochondrial dysfunction in the elderly: possible role in insulin resistance. Science 300:1140-1142

10. Thamer C, Machann J, Bachmann O et al (2003) Intramyocellular lipids: anthropometric determinants and relationships with maximal aerobic capacity and insulin sensitivity. J Clin Endocrinol Metab 88:1785-1791

11. Weiss R, Dufour S, Groszmann A et al (2003) Low adiponectin levels in adolescent obesity: a marker of increased intramyocellular lipid accumulation. J Clin Endocrinol Metab 88:2014-2018

12. van Loon LJ, Koopman R, Manders R, van der Weegen W, van Kranenburg GP, Keizer HA (2004) Intramyocellular lipid content in type 2 diabetes patients compared with overweight sedentary men and highly trained endurance athletes. Am J Physiol Endocrinol Metab 287:E558-E565

13. Kuipers H, Verstappen FT, Keizer HA, Geurten P, van Kranenburg G (1985) Variability of aerobic performance in the laboratory and its physiologic correlates. Int J Sports Med 6:197-201

14. DeFronzo RA, Tobin JD, Andres R (1979) Glucose clamp technique: a method for quantifying insulin secretion and resistance. Am J Physiol 237:E214-E223

15. Naressi A, Couturier C, Devos JM et al (2001) Java-based graphical user interface for the MRUI quantitation package. Magma 12:141-152
16. Newcomer BR, Boska MD (1999) $\mathrm{T} 1$ measurements of ${ }^{31} \mathrm{P}$ metabolites in resting and exercising human gastrocnemius/soleus muscle at 1.5 Tesla. Magn Reson Med 41:486-494

17. Schrauwen-Hinderling VB, Schrauwen P, Hesselink MK et al (2003) The increase in intramyocellular lipid content is a very early response to training. J Clin Endocrinol Metab 88:1610-1616

18. Lexell J, Henriksson-Larsen K, Sjostrom M (1983) Distribution of different fibre types in human skeletal muscles. 2. A study of cross-sections of whole m. vastus lateralis. Acta Physiol Scand 117:115-122

19. Vanhamme L, van den Boogaart A, Van Huffel S (1997) Improved method for accurate and efficient quantification of MRS data with use of prior knowledge. J Magn Reson 129:35-43

20. Meyer RA, Foley JM (1996) Cellular processes integrating the metabolic responses to exercise. In: Rowell LB, Shepherd JT, eds. Handbook of physiology. American Physiological Society, New York, pp 841-869

21. Kemp GJ, Radda GK (1994) Quantitative interpretation of bioenergetic data from ${ }^{31} \mathrm{P}$ and ${ }^{1} \mathrm{H}$ magnetic resonance spectroscopic studies of skeletal muscle: an analytical review. Magn Reson Q 10:43-63

22. Sahlin K, Harris RC, Hultman E (1979) Resynthesis of creatine phosphate in human muscle after exercise in relation to intramuscular $\mathrm{pH}$ and availability of oxygen. Scand J Clin Lab Invest 39:551-558

23. Kemp GJ, Taylor DJ, Radda GK (1993) Control of phosphocreatine resynthesis during recovery from exercise in human skeletal muscle. NMR Biomed 6:66-72

24. Arias-Mendoza F (2004) In vivo magnetic resonance spectroscopy in the evaluation of mitochondrial disorders. Mitochondrion 4:491-501

25. Mattei JP, Bendahan D, Cozzone P (2004) P-31 magnetic resonance spectroscopy. A tool for diagnostic purposes and pathophysiological insights in muscle diseases. Reumatismo 56:9-14

26. Larson-Meyer DE, Newcomer BR, Hunter GR, Joanisse DR, Weinsier RL, Bamman MM (2001) Relation between in vivo and in vitro measurements of skeletal muscle oxidative metabolism. Muscle Nerve 24:1665-1676

27. De Feyter HM, Praet SS, van Loon LJ, Prompers JJ, Nicolay K (2006) A comparison of different methodologies to study skeletal muscle mitochondrial function in Type 2 diabetes patients. In: International Society of Magnetic Resonance in Medicine Seattle, p 483 (Abstract)

28. Stump CS, Short KR, Bigelow ML, Schimke JM, Nair KS (2003) Effect of insulin on human skeletal muscle mitochondrial ATP production, protein synthesis, and mRNA transcripts. Proc Natl Acad Sci USA 100:7996-8001

29. Sreekumar R, Halvatsiotis P, Schimke JC, Nair KS (2002) Gene expression profile in skeletal muscle of type 2 diabetes and the effect of insulin treatment. Diabetes 51:1913-1920

30. Morino K, Petersen KF, Dufour S et al (2005) Reduced mitochondrial density and increased IRS-1 serine phosphorylation in muscle of insulin-resistant offspring of type 2 diabetic parents. J Clin Invest 115:3587-3593

31. Schrauwen P, Mensink M, Schaart G et al (2005) Reduced skeletal muscle UCP3 protein content in pre-diabetic subjects and type 2 diabetic patients: restoration by rosiglitazone treatment. J Clin Endocrinol Metab

32. Taylor DJ, Crowe M, Bore PJ, Styles P, Arnold DL, Radda GK (1984) Examination of the energetics of aging skeletal muscle using nuclear magnetic resonance. Gerontology 30:2-7 